\title{
CHARACTERIZATION OF REFLEXIVE BANACH SPACES WITH NORMAL STRUCTURE
}

\author{
Milan R. Tasković ${ }^{*}$
}

\begin{abstract}
This paper presents a characterization of reflexive Banach spaces with normal structure in term of fixed point for diametral contractive mappings.
\end{abstract}

\section{Introduction and history}

Kakutani [10] and Markoff [14] have shown that if a commutative family of continuous affine maps of a linear topological space into itself leaves some nonempty compact convex subset invariant, then the family has a common fixed point in this invariant subset. Precisely, this statement was proved in 1936 by Markoff with the aid of the Schauder-Tychonoff fixed point theorem, till S. Kakutani in 1938 found a direct elementary proof and demonstrated the importance of the result by giving a number of applications; he also showed that this result implies the Hahn-Banach principle in linear functional analysis.

Fixed-point theorems for families of continuous linear or affine transformations also have been obtained by Day [5] and Göhde [7].

Fixed-point theorems for families of nonexpansive mappings established by De Marr [6] (for Banach spaces), F. Browder [4] (for uniformly convex spaces) do not apply in this class of sets, and T. Lim [13] (for reflexive Banach spaces).

AMS (MOS) Subject Classification 1991. Primary: 47H10,05A15. Secondary: 54H25.

Key words and phrases: Reflexive Banach spaces, normal structure, diametral sequences, Brodskij-Milman theorem, Šmulian property, characterization of normal structure, diametral contractive mappings, fixed points.

*Research supported by Science Fund of Serbia under Grant 1457. 
Let $X$ be a Banach space. A mapping $T$ of a subset $K$ of $X$ into $X$ is called diametral contraction on $K$ if

$$
\|T x-T y\| \leq \sup \{\|x-z\|: z \in K\}
$$

for all $x, y \in K$. In a former paper Tasković [17] investigated a nonempty commutative family $\mathcal{F}$ of diametral contractive mappings of a nonempty compact convex subset $K$ into itself, and showed that the family $\mathcal{F}$ has a common fixed point in $K$.

We notice, by interchanging $x$ and $y$ we see that condition (D) may be written in a widening and broad form with

$$
\|T x-T y\| \leq \max \{\sup (\|x-z\|: z \in K), \sup (\|y-z\|: z \in K)\}
$$

for all $x, y \in K$. The condition (Da) is not equivalent to the condition (D), since has been notice P. Kenderov [18].

Open problem 1. If $K$ is a nonempty, bounded, closed and convex subset of a reflexive Banach space $X$ and if $T: K \rightarrow K$ with the property (Da) does $T$ have a fixed point in $K$ ?

On the other hand, parallely with the preceding results, first a general fixed point result for isometries was obtained by Brodskij-Milman [3].

For this result the concept of normal structure, due to Brodskij - Milman [3], plays a key role.

For $S \subset X$ we denote the diameter of $S$ by $\delta(S)$. A point $x \in S$ is a diametral point of $S$ provided

$$
\sup \{\|x-y\|: y \in S\}=\delta(S) .
$$

A convex set $K \subset X$ is said to have normal structure if for each bounded convex subset $H$ of $K$ which contains more than one point, there is some point $x \in H$ which is not a diametral point of $H$. Compact convex sets possess normal structure obtained by Brodskij - Milman [3].

Kirk [12] proved that if $K$ is a nonempty weakly compact convex subset of a Banach space and if $K$ has normal structure, then every nonexpansive selfmap $T$ of $K$ has a fixed point. Later, Belluce - Kirk [1] extend this statement by showing that any finite family of commuting nonexpansive self-mappings of such a set $K$ always has a common fixed point.

In this paper we proved that every reflexive Banach space with normal structure possesses the fixed point property for diametral contractive mappings.

It is natural to ask whether this result remains true for reflexive Banach spaces without normal structure. As an answer for this question we prove that every normed space possessing the fixed point property for dametral contractive mappings has normal structure. 
In connection with this, Brodskij-Milman [3] obtained the following characterization of normed spaces with normal structure, that a normed space has normal structure if and only if it contains no diametral sequences.

\section{A fixed point theorem}

In this part we extend Kirk's theorem and we describe a result for the existence of fixed points which generalize several known results.

We are now in the position of proving the following statement use a characterization of reflexivity due to Šmulian and the preceding concept of Brodskij and Milman.

Theorem 1. Let $K$ be a nonempty, bounded, closed and convex subset of a reflexive Banach space $X$, and suppose that $K$ has normal structure. If $T$ is a diametral contractive mapping of $K$ into itself, then $T$ has a fixed point in $K$.

Proof. Let $\mathcal{F}$ denote the collection of all nonempty closed and convex subsets of $K$, each of which is mapped into itself by $T$. Obviously, $\mathcal{F}$ is nonempty. Now we define a partial ordering on $\mathcal{F}$ by the set inclusion relation. By Šmulian's theorem ( $X$ is reflexive Banach space if and only if every bounded desceding transfinite sequence of nonempty closed convex subset of $X$ have a nonempty intersection) and Zorn's lemma $\mathcal{F}$ has a minimal element which we denote by $S$. Here, $S$ being minimal with the respect to being nonempty, bounded, closed convex and invariant under $T$. We complete the proof by showing that $S$ consists of a single point.

If $S$ contains only one element, then that element is a fixed point of $T$. If not, let $S$ contain more than one element, then it follows from the normal structure of $K$ that there exists $c \in S$ such that

$$
\sup \{\|c-y\|: y \in S\}:=\rho<\delta(S) .
$$

Thus and from (D) we obtain the following inequality

$$
\|T c-T y\| \leq \sup \{\|c-y\|: y \in S\}=\rho .
$$

Hence, $T(S)$ is contained in the closed ball $B$ with $T c$ as center and $\rho$ as radius. Also, $S \cap B$ is invariant under $T$, therefore, by the minimality of $S$ it follows that $S \subset M$, i.e., $\|T c-x\| \leq \rho$ for every $x \in S$. Hence, we have

$$
\sup \{\|T c-x\|: x \in S\} \leq \rho .
$$

Consider the set

$$
F=\{z \in S: \sup [\|z-x\|: x \in S] \leq \rho\} .
$$


Obviously $F$ is closed, convex and nonempty $(T c \in F)$. Again if $z \in F$, then $z \in S$ and hence $T z \in F$ by (1). Hence $F$ is invariant under $T$. Also, we have

$$
\delta(F) \leq \sup \{\|x-y\|: x \in F, y \in S\} \leq \rho<\delta(S),
$$

by hypothesis and the preceding facts. Thus $F$ is a proper subset of $S$, which contradicts the minimality of $S$. Hence $S$ has only one element which is a fixed point of $T$. The proof is complete.

\section{Characterization of reflexive spaces with normal structure}

It is natural to ask whether the preceding Theorem 1 remains true for reflexive Banach spaces without normal structure. In this part we answer this question in the negative. We prove that Theorem 1 is the best possible in sense that every normed space possesing the fixed point property for diametral contractive mappings has normal structure.

First characterization of normed spaces with normal structure was given by Brodskij - Milman [3] in terms of diametral sequences. A characterization was given by Kassay [11].

A bounded sequence $\left\{x_{n}\right\}_{n \in \mathbb{N}}$ is diametral if it is non-constant and if $\delta\left(x_{n}, \operatorname{conv}\left\{x_{1}, \ldots, x_{n-1}\right\}\right) \rightarrow \delta\left(\left\{x_{n}\right\}_{n \in \mathbb{N}}\right)$, where conv $A=$ convex hull of $A$.

Let $X$ be a normed space and we define $\mathcal{K}$ as a collection of all closed, convex, bounded subsets of $X$, each of them containing more than one element.

One says that $X$ has normal structure if every $K \in \mathcal{K}$ contains a point which is not diametral point of $K$.

In connection with the preceding, a mapping $T: K \rightarrow K$ (for $K \in \mathcal{K}$ ) is said to be diametral contractive if

$$
\|T x-T y\| \leq \sup \{\|x-z\|: z \in Y\}
$$

for every $Y \in \mathcal{K}$ with $Y \subset K$ and for all $x, y \in Y$. A normed space $X$ is said to have diametral fixed point property if every diametral contractive map $T: K \rightarrow K$ (for an arbitrary $K \in \mathcal{K}$ ) has a fixed point.

With the help of the preceding Theorem 1 we now obtain the main result of this paper in the following form.

Theorem 2. (Characterization of normal structure). A reflexive $B a-$ nach space has normal structure if and only if it possesses the diametral fixed point property.

Proof. Since the condition of this statement is known to be necessary for normal structure from Theorem 1, we have only to show that it is sufficient. 
Supposing the contrary, there is a reflexive space (or only a normed space) $X$ without normal structure and with diametral fixed point property.

Then from Brodskij - Milman's result in [3], $X$ contains a diametral sequence $\left\{x_{n}\right\}_{n \in \mathbb{N} \cup\{0\}}$. Thus for the set $K=\overline{\operatorname{conv}\left(\left\{x_{n}\right\}\right)} \in \mathcal{K}$ we obtain $\delta(K)=\delta\left(\left\{x_{n}\right\}\right)$. On the other hand, from the definition of diametral sequences, we have with $n \rightarrow \infty$ that

$$
\left\|x-x_{n}\right\| \rightarrow \delta(K) \text { for every } x \in \operatorname{conv}\left(\left\{x_{n}\right\}\right) .
$$

This means, because $\delta(K)>0$, that the sequence $\left\{x_{n}\right\}_{n \in \mathbb{N} \cup\{0\}}$ has no constant subsequence.

We define a mapping $T$ from $K$ into itself by

$$
T(x)=\left\{\begin{array}{lll}
x_{0} & \text { if } \quad x \notin\left\{x_{n}\right\}_{n \in \mathbb{N} \cup\{0\}}, \\
x_{n+1} & \text { if } \quad x=x_{n} \quad(\text { for } n \in \mathbb{N} \cup\{0\}) .
\end{array}\right.
$$

Thus we have defined a function $T$ on $K$ to $K$. Now, for $K \in \mathcal{K}$ we have $T(K)=\left\{x_{n}\right\}_{n \in \mathbb{N} \cup\{0\}} \subset K$, so $T$ does not have a fixed point.

On the other hand, for $Y \in \mathcal{K}$ and $Y \subset K$ let $\varepsilon$ be an arbitrary positive number and let us choose an element $z \in \operatorname{conv}\left(\left\{x_{n}\right\}\right)$ such that $\|z-x\|<\varepsilon$ for $x \in K$. Thus we obtain the following inequalities

$$
\begin{gathered}
\delta(K) \geq \sup \{\|x-y\|: y \in Y\} \geq \sup \{\|z-y\|: y \in Y\}-\|z-x\|> \\
>\sup \left\{\left\|z-x_{n(k)}\right\|: \text { for } k \in \mathbb{N}\right\}-\varepsilon \geq \delta(K)-\varepsilon,
\end{gathered}
$$

where $\left\{x_{n(k)}\right\}_{k \in \mathbb{N}}$ is a subsequences in $Y$ of the sequence $\left\{x_{n}\right\}_{n \in \mathbb{N} \cup\{0\}}$. Hence, directly, we have

$$
\delta(K)=\sup \{\|x-y\|: y \in Y\} \geq \sup \{\|T x-T y\|: y \in Y\} \geq\|T x-T y\|
$$

for all $x, y \in Y$. Thus $T$ satisfies (D') and does not have a fixed point. The proof is complete.

Some remarks. We note that from the preceding proof of Theorem 2 , sufficient in this statement is a special case of more general the following result: Every normed space with the diametral fixed point property has normal structure. The proof of this fact is equal with the preceding proof of sufficiently in Theorem 2.

Open problem 2. Let $K$ be a nonempty weakly compact convex subset of Banach space and assume that $K$ has normal structure. If $\mathcal{K}$ an arbitrary family of commuting diametral contractive maps from $K$ into itself, does $\mathcal{K}$ have a common fixed point?

\section{References}

[1] L.P. Belluce and W.A. Kirk: Fixed point theorems for families of contraction mappings, Pacific J. Math., 18(1966), 213-217. 
[2] L.P. Belluce and W.A. Kirk: Nonexpansive mappings and fixed-points in Banach spaces, Illinois J.Math., 11(1967), 474-479.

[3] M.S. Brodskij and D.P. Milman: On the center of a convex set, Dokl. Akad. Nauk SSSR (N.S.), 59 (1948), 837-840.

[4] F.E. Browder: Nonexpansive nonlinear operators in a Banach space, Proc. Nat. Acad. Sci., 54 (1965), 1041-1044.

[5] M.M. Day: Fixed-point theorems for compact convex sets, J. Math., 5 (1961), 585-590.

[6] R. De Marr: Common fixed-points for commuting contraction mappings, Pacific J. Math., 13 (1963), 1139-1141.

[7] D. Göhde: Über Fixpunkte bei stetigen Selbstabbildungen mit kompakten Iterierten, Math. Nach., 28 (1964), 45-55.

[8] D. Göhde: Zum Princip der kontraktiven Abbildung, Math. Nachr., 30 (1965), 251-258.

[9] K. Goebel, W.A. Kirk and T.N. Shimi: A fixed point theorem in uniformly convex spaces, Bollettino U.M.I., 7 (1973), 67-75.

[10] S. Kakutani: Two fixed-point theorems concerning bicompact convex sets, Proc. Imp. Acad. Tokyo, 14 (1938), 242-245.

[11] G. Kassay: A characterization reflexive Banach spaces with normal structure, Bollettino U.M.I., 5-A (1986), 273-276.

[12] W.A. Kirk: A fixed point theorem for mappings which do not increase distances, Amer. Math. Monthly, 72 (1965), 1004-1006.

[13] T.C. Lim: A fixed point theorem for families of nonexpansive mappings, Pacific J. Math., 53(1974), 487-493.

[14] A. Markoff: Quelques théorémes sur les esembles Abèliens, Doklady Akad. Nauk SSSR (N.S.), 10 (1936), 311-314.

[15] V. Šmulian: On the principle of inclusion in the space of type (B), Math. Sb. (N.S.), 5 (1939), 327-328.

[16] M.R. Tasković: Reflexive Banach space and fixed point theorems, Publ. Inst. Math. (Beograd), 34 (1976), 243-247.

[17] M.R. Tasković: Some results in the fixed point theory-II, Pub. Inst. Math. (Beograd), 41 (1980), 249-258.

[18] P. Kenderov: Private communication, Sofia, September 25 th, 1997.

Matematički fakultet 11000 Beograd, P.O. Box 550 Yugoslavia

Received January 27, 2001. 